\section{Psicologia Escolar \\ e Educacional}

\section{ARTí́cULO}

DOI: http://dx.doi.org/ 10.1590/2175-35392021220278

Localizador - e220278

\title{
PENSAMIENTO METACOGNITIVO, CRÍTICO Y CREATIVO EN CONTEXTOS EDUCATIVOS: CONCEPTUALIZACIÓN Y SUGERENCIAS DIDÁCTICAS
}

\author{
Laura Espinoza Pastén ${ }^{1} \mathbb{D}$
}

\begin{abstract}
RESUMEN
El presente artículo aborda los conceptos de pensamiento metacognitivo, crítico y creativo, planteando una revisión desde los aportes desde la psicología y desde la neurociencia cognitiva, y su impacto tanto en el aprendizaje como en el rendimiento académico. Como primer objetivo, se plantea una conceptualización de los procesos de pensamiento metacognitivo, crítico y creativo según la literatura clásica y actual. En segundo lugar, se sugieren algunas acciones didácticas a los profesionales de la educación para estimular el desarrollo de cada uno de los tipos de pensamiento. Como resultado de la revisión y la reflexión, se concluye que el desarrollo del pensamiento es clave para hacer más eficientes los procesos de enseñanza y aprendizaje, puesto que el estudiante adquiere un rol activo y autonomía en la construcción del conocimiento y el desarrollo de habilidades, que trascienden a las diferentes esferas del desarrollo humano.
\end{abstract}

Palabras clave: Metacognición; pensamiento crítico; creatividad.

\section{Metacognitive, critical and creative thinking in educative contexts: conceptualization and didactic suggestions}

\begin{abstract}
The present article deals with the concepts of metacognitive, critical and creative thinking, proposing a revision from the contributions from psychology and cognitive neuroscience, and its impact on both learning and academic performance. As a first objective, a conceptualization of metacognitive, critical and creative thought processes according to classical and current literature is proposed. Second, some didactic actions are suggested to education professionals to stimulate the development of each type of thinking. As a result of the review and reflection, it is concluded that the development of thinking is key to making the teaching and learning processes more efficient, since the student acquires an active role and autonomy in the construction of knowledge and the development of skills, that transcend the different spheres of human development.
\end{abstract}

Keywords: Metacognition; critical thinking; creativity.

\section{Pensamento metacognitivo, crítico e criativo em contextos educativos: conceitualização e sugestões didáticas}

\begin{abstract}
RESUMO
O presente artigo aborda os conceitos de pensamento metacognitivo, crítico e criativo, abordando uma revisão desde os aportes da psicologia e da neurociência cognitiva, e seu impacto tanto na aprendizagem como no rendimento acadêmico. Como primeiro objetivo, aborda-se uma conceitualização dos processos de pensamento metacognitivo, crítico e criativo segundo a literatura clássica e atual. Em segundo lugar, sugerem-se algumas ações didáticas aos profissionais da educação para estimular o desenvolvimento de cada um dos tipos de pensamento. Como resultado da revisão e da reflexão, conclui-se que o desenvolvimento do pensamento é a chave para fazer mais eficientes os processos de ensino e aprendizagem, posto que o estudante adquire um papel ativo e autonomia na construção do conhecimento e o desenvolvimento de habilidades, que transcendem as diferentes esferas do desenvolvimento humano.
\end{abstract}

Palavras-chave: Metacognição; pensamento crítico; criatividade.

\footnotetext{
${ }^{1}$ Departamento de Educación y colaboradora IESED - Universidad de Los Lagos - Osorno - Chile; Laura.espinoza@ulagos.cl
} 


\section{INTRODUCCIÓN}

En el actual contexto educativo nacional e internacional se plantea que el aprendizaje de los estudiantes debiera enfocarse, además de los contenidos, en el desarrollo de habilidades cognitivas. Sin embargo, es posible que los docentes de aula tiendan a enfatizar el aprendizaje del contenido disciplinar por sobre la estimulación explícita del pensamiento necesario para aprender. Frente a esta situación, surge la inquietud de las formas en que la estimulación del pensamiento puede ser considerada explícitamente en los contextos educacionales, con el objetivo de potenciar el aprendizaje de calidad.

El presente trabajo presenta elementos teóricos y reflexivos sobre el pensamiento metacognitivo, crítico y creativo desde una perspectiva cognoscitiva. A partir de estos aportes, se proponen algunas sugerencias específicas y posibles de llevar a cabo en diversas situaciones de aprendizaje, sin diferenciar en la edad, nivel educativo o características del estudiantado. Para finalizar, se presentan algunas conclusiones que refuerzan la importancia del desarrollo del pensamiento como una forma de potenciar los aprendizajes en el contexto educacional.

\section{Pensamiento Metacognitivo}

Los aportes teóricos sobre la metacognición parten históricamente desde el psicólogo de origen canadiense John Flavell, en la década de los años setenta. En sus publicaciones clásicas, define este concepto como pensar sobre el pensar, permitiéndose un monitoreo constante de este proceso.

El pensamiento metacognitivo se puede describir como aquella capacidad de inspeccionar nuestras propias actividades mentales, reconociendo en ellas las acciones secuenciadas que realizamos. Es tener conciencia sobre nuestro procesamiento mental teniendo control sobre éste (Lacón \& Ortega, 2008). Por tanto, la metacognición ayuda a hacer una autosupervisión de la actividad mental, tomando decisiones al respecto para mejorarla y controlar elementos que pueden favorecerla o entorpecerla (Sinatra \& Taasoobshirazi, 2017).

Desde una perspectiva psicológica, el pensamiento metacognitivo consta de dos dimensiones o componentes principales, según Soto (2002): conocimiento cognitivo y regulación cognitiva. Por un lado, la dimensión de conocimiento cognitivo se refiere a la información tanto conceptual como experiencial que se tiene y se cree sobre factores que pueden influir en el desempeño de una tarea, por ejemplo: conocer de sí mismo las fortalezas o los recursos cognitivos. El autor menciona que el conocimiento puede referirse tanto a la persona que puede ser uno mismo u otro con los que se interactúa, a la naturaleza de la tarea ante la cual se enfrenta el estudiante y la dificultad que representa, y a la efectividad de las estrategias que se emplean para enfrentarla y resolverla. Por otro lado, respecto de la regulación de los procesos cognitivos, Soto plantea que se hacen partícipes algunas funciones específicas mentales, tales como la planificación, supervisión y evaluación.

En las últimas dos décadas han iniciado los aportes desde investigadores de las neurociencias, que evidencian algunas bases y correlatos neurales que sustentan los procesos de metacognición. En este sentido, se vincula con bases neurales de memoria y de toma decisiones, presentes sobre todo en la zona dorsolateral y anterior de la corteza prefrontal del cerebro, y sus conexiones con zonas más internas (Fleming \& Dolan, 2012). También se han vinculado algunas funciones ejecutivas para el procesamiento metacognitivo. Procesos ejecutivos tales como la autorregulación, inhibición y atención ejecutiva que son parte de complejas redes neurales asociadas a la corteza prefrontal del cerebro, colaborarían para el funcionamiento de la metacognición (Shimamura, 2000). Por otro lado, se ha investigado que elementos afectivos pueden modular la ejecución de la metacognición, tanto en situaciones o tareas de índole social y afectiva o cognitiva-académica. Molenberghs, Trautwein, Böckler, Singer y Kanske (2016), en su investigación, hallaron que la auto confianza afecta la ejecución metacognitiva, llegando a disminuir la precisión si es excesiva, o gatillando incertidumbre si la autoconfianza es baja. Por tanto, redes cerebrales complejas e interconectadas son base de la metacognición.

Algunos autores plantean que el pensamiento metacognitivo es importante para el aprendizaje a lo largo de la vida escolar y académica. Durante la primera escolaridad, sería un proceso importante para el alcance de aprendizajes tales como la producción escrita (Aguirre, 2016) y como la lectura Veenman (2015). Durante la educación primaria y secundaria, el estudio de Tanikawa y Boruchovitch (2016) encontraron que los estudiantes más jóvenes tienen mejores habilidades de supervisión que los más mayores, e incluso presentan mejor rendimiento académico. En educación secundaria según estudios, la metacognición muestra también su implicancia en procesos de aprendizaje de la comprensión lectora (Karbalaei, 2011), sobre todo para que el estudiante pueda dar respuestas adecuadas a las preguntas que exigen comprensión de tipo inferencial (Soto et al., 2019). En educación superior, el pensamiento metacognitivo impacta la calidad de los aprendizajes en las áreas, por ejemplo, del aprendizaje de otros idiomas (Karbalaei, 2011). Asimismo, en la mejora de la comprensión lectora de futuros profesores, quienes deben estar preparados para enseñar dichas 
estrategias a sus futuros estudiantes (Iwai, 2016). A partir de los estudios mencionados, se evidencian las relaciones entre la metacognición y el aprendizaje en contextos educacionales.

\section{Pensamiento Crítico}

Desde una perspectiva cognitiva el pensamiento crítico se refiere al proceso de reflexión, evaluación de razonamientos y posterior toma de decisiones para resolver problemas. Para Nieto y Saiz (2011) el pensamiento crítico debe efectuarse de forma intencional o deliberada, para llegar así a un juicio con base en la razón que orientará las posteriores acciones, decisiones e incluso creencias. Además de lo cognitivo, el pensamiento crítico tiene un impacto en la forma en que la persona se enfrenta y se integra a una sociedad, cómo es capaz de reflexionar sobre ella y a su vez, ser un sujeto activo en ella (Jiménez-Aleixandre, 2010). Por tanto, es importante su estimulación en etapas tempranas, pues es necesario para enfrentar problemas o situaciones como ciudadanos (Gormley, 2017).

Uno de los primeros autores en publicar sobre el pensamiento crítico fue Ennis. El autor planteaba que el pensamiento crítico tenía tres dimensiones: dimensión lógica (relación de los significados de las verbalizaciones), dimensión criterial (opinar sobre estas verbalizaciones y juzgarlas) y dimensión pragmática (relación juicio-decisión y su impacto en el contexto). En sus trabajos más actuales y vigentes para la teoría del pensamiento crítico, Ennis (2011) plantea la presencia tanto de habilidades como de disposiciones, necesarios para llevar a cabo el pensamiento crítico. Respecto de las habilidades, Ennis propone las siguientes agrupadas en categorías:

1. Habilidades de aclaración básica. Focalizarse en la pregunta, analizar argumentos, preguntar sobre aclaraciones respecto de las preguntas.

2. Habilidades de base para la toma de una decisión. Juzgar la credibilidad de una fuente, observar y juzgar lo que se reporta por medio de la observación.

3. Habilidades de inferencia. Deducir y juzgar la propia deducción, realizar inferencias específicas, generar y juzgar juicios de valor propios y de otros.

4. Habilidades de clarificación avanzada. Definir términos y juzgar las definiciones propias y de otros, asignar supuestos que no se encuentran declarados.

5. Habilidades de suposición e integración. Tener en cuenta y razonar a partir de premisas, suposiciones, razones y posiciones con los que no se está de acuerdo o causan escepticismo, sin que este estado dubitativo interfiera en el pensar.
6. Habilidades auxiliares. Estas habilidades son complementarias y permiten optimizar la ejecución del pensamiento crítico. Entre ellas encontramos: proceder de forma organizada ante la situación, y ser sensible al nivel de conocimiento, a los sentimientos y grado de sofisticación de otros.

En relación a las disposiciones, las cuales se definen como el estado interno del aprendiz para llevar algo a cabo, Ennis plantea las siguientes categorías:

1. Cuidar que sus decisiones se encuentren debidamente fundamentadas y sus creencias sean verosímiles, dentro de sus posibilidades. Para ello, el pensador crítico estará dispuesto a buscar opciones o vías alternativas posibles, a buscar puntos de vista diferentes al propio, a mantenerse bien informado, a aprobar posiciones diferentes siempre que se encuentren bien justificadas, y a hacer uso de sus propias habilidades de pensamiento crítico.

2. Cuidar la comprensión y la presentación clara de una postura, sea propia o de otros. Para lograrlo, estará dispuesto a escuchar opiniones y argumentos ajenos, a ser claro al transmitir información sin alterar su significado, a mantener el enfoque en el cuestionamiento o conclusión, a buscar y entregar argumentos, a visualizar la situación de forma integral, y a ser consciente reflexionando sobre las propias creencias que se tienen de base.

Al igual que la metacognición, el pensamiento crítico también tiene implicancia en diferentes disciplinas según estudios. En educación secundaria, la literatura sugiere incluir estrategias didácticas para su estimulación y la formación de docentes quienes deben llevarlas a cabo (Moreno \& Velázquez, 2017). A nivel de educación universitaria, se ha hallado según la literatura que esta tiene un efecto en el desarrollo del pensamiento crítico (Huber \& Kuncel, 2016). También se ha verificado la importancia de evaluar este proceso, para ser potenciado y mejorar el rendimiento en la formación profesional en carreras de la salud (Ospina, Brand, \& Aristizabal, 2017).

\section{Pensamiento Creativo}

El pensamiento creativo es la actividad mental en la que se genera nueva información no evidente con un fin determinado. Esta información nueva es una propuesta de un procedimiento o de un elemento inexistente 0 desconocido (Abraham, 2018), independiente de que ya exista o haya sido creado por otros previamente. Va más allá del arte. Es la capacidad investigativa original, 
que aporta lo novedoso, lo práctico, lo útil (Belmonte, 2013). Asimismo, permite un abordaje innovador ante alguna situación o conflicto dado, al generar respuestas e ideas divergentes para resolverlo (Rawlinson, 2017).

La investigación científica del pensamiento creativo inició en la década de los cincuenta, momento en que se formularon desde la psicología las primeras concepciones sobre creatividad, las primeras propuestas para su medición así como incipientes estrategias para su estimulación. En los años setenta, aparecen con renombre autores tales como Torrance y De Bono, quienes desarrollaron algunos elementos conceptuales, plantearon instrumentos para su evaluación y propusieron programas específicos para su estimulación (Prieto, López, Ferrándiz, \& Bermejo, 2003).

Torrance, en su trabajo clásico de 1974 (citado en López \& Navarro, 2008) señala que la creatividad permite detectar dificultades, espacios en blanco, disonancias y errores; lo cual lleva al planteamiento de hipótesis y búsqueda de soluciones de manera recursiva. Además, el autor plantea que el pensamiento creativo constaría de componentes tales como la fluidez o capacidad de producir ideas, flexibilidad o capacidad de ajuste, originalidad y elaboración. Por su parte, De Bono plantea que para formular soluciones creativas o propuestas que sean realmente originales y útiles es necesario manejar la información, ciertas bases o parámetros concretos desde los cuales nace la creación o propuesta inédita (De Bono, 2012).

Sobre la estimulación del pensamiento creativo, en jóvenes y adultos se ha demostrado que es estimulable independiente de la edad (Madore, Jing, \& Schacter, 2016). Específicamente en los contextos educativos, en estudiantes de educación secundaria se sugiere el trabajo por medio de la resolución de situaciones problemáticas (Birgili, 2015), cuyo tratamiento debe llevarse a cabo haciendo planteamiento de preguntas abiertas, que hagan al estudiantado cuestionarse (Borjas \& De La Peña, 2009). La escuela sería un lugar apropiado para estimular la creatividad, sobre todo en educación primaria y secundaria ya que posteriormente se ve un descenso de la manifestación de los componentes del pensamiento creativo (Kim, 2011).

Durante las últimas dos décadas se han gestado recientes aportes sobre el pensamiento creativo desde la neurociencia cognitiva. Respecto de este tema, es importante comprender que no existe una zona neural específica de la creatividad, sino más bien redes interconectadas complejas que permiten que se lleve a cabo este proceso de nivel superior (Beaty, Benedeck, Silvia, \& Schacter, 2016; Beaty et al., 2018). Por una parte, se señala que existe una predominancia del hemisferio derecho al ejecutar tareas creativas de diversa índole (Mihov, Denzler, \& Förster, 2010). Por otra parte, el modelo propuesto por Flaherty en 2005 explica la creatividad en red, donde da importancia a la zona prefrontal del cerebro (base de las funciones ejecutivas), y las conexiones existentes con los lóbulos temporales y zonas del sistema límbico. Es decir, las ideas creativas surgen considerando funciones complejas como las ejecutivas: generación de ideas, hipótesis, memoria de trabajo, planificación, autorregulación, además de procesos de memoria, lenguaje y emociones (sistema límbico). Respecto de las emociones, y así como lo señalan otros estudios más recientes (Perchtold et al., 2018) Flaherty planteó en su modelo que el componente emocional de la red sería un regulador de esta, sobre todo de la actividad mental en la zona prefrontal. El modelo de Flaherty sigue vigente y es coherente con la literatura más reciente (Beaty et al., 2018; Green, Cohen, Raab, Yedibalian, \& Gray, 2015). Por último, la evidencia también señala la participación de la conciencia con un rol fundamental, pues estos procesos también tienen sus bases neurales principales en la zona prefrontal y es allí donde se generarían las ideas (Dietrich, 2004).

\section{Pensamiento metacognitivo, crítico y creativo en la educación actual}

En los contextos educacionales, parece ser que existe claridad en que para aprender es importante pensar. Sin embargo, no parece que todos los maestros enseñen a los alumnos las habilidades del pensamiento de manera explícita e intencionada. Por lo mismo, no todos los estudiantes logran ver mejoras al ejecutar dichas habilidades durante los procesos de aprendizaje.

Respecto del pensamiento metacognitivo, se ha evidenciado su vinculación con el rendimiento académico y en diversas áreas de aprendizaje (Aguirre, 2016; Karabalei, 2011; Soto et al., 2019; Tanikawa y Boruchovitch, 2016; Veenman, 2015). La literatura plantea que es necesario generar investigación educativa sobre estrategias de enseñanza efectivas para el desarrollo del pensamiento, sobre todo de la metacognición y la autorregulación para el aprendizaje en el contexto escolar. El desarrollo del pensamiento metacognitivo requiere de la implementación de estrategias por parte del aprendiz que, previamente, son intencionadas y explicitadas en el entorno de aprendizaje (Soto et al., 2019). Sin embargo, no se requiere la realización de un programa estructurado complementario o anexo a la enseñanza del currículo común, más bien es preciso desarrollarlas de forma transversal en la educación dentro del aula (Monereo, 2001). En síntesis, el pensamiento metacognitivo aporta a la autonomía del aprendizaje de los estudiantes, y a su transferencia a otros contextos de la vida por lo cual debe ser intencionado transversalmente en diferentes momentos y contextos.

Respecto del pensamiento crítico y como se mencionó con anterioridad, se relaciona también con 
procesos ejecutivos como la toma de decisiones para la solución de problemas (Gormley, 2017), ante la cual debemos efectuar una valoración de los elementos o componentes, respecto de: sus fortalezas y debilidades, con objetividad, apreciar su funcionalidad, eficiencia, aportes y proyección en el tiempo. Todos estos procesos generan una carga de elaboración cognoscitiva a las actividades que impliquen explícitamente el pensamiento crítico. No obstante, los estudiantes incluso a nivel universitario, muestran un bajo desempeño en este tipo de pensamiento (Tenías, 2012). Por lo cual, sería necesario llevar estrategias didácticas prácticas al salón de clases y formar al profesorado en ello (Moreno \& Velásquez, 2017), para ejecutarlo de manera concreta y no solamente efectuar su estudio a nivel teórico. Esto sería relevante, pues el pensamiento crítico permite una transformación continua del pensar y la resolución consecutiva de problemas variados tanto en el contexto escolar-académico como en la vida (Elder \& Paul, 2008).

En relación al pensamiento creativo, en la educación actual se sigue vinculando a procesos de creación artística. Las estrategias para el desarrollo del pensamiento creativo e incluso las pruebas que se emplean aún se basan en paradigmas clásicos de la década de los años setenta, siendo importante su ajuste desde los actuales aportes de la psicología y la neurociencia cognitiva. Se requiere que estos aportes actualizados, además, permeen al contexto educativo para educar aprendices más reflexivos y que presenten mayor interés en su proceso de creación o enfrentamiento de la situación problema (Birgili, 2015; Pacheco, 2003).

Orientaciones didácticas para la estimulación del pensamiento metacognitivo, crítico y creativo en el contexto educativo

La metacognición se activa cuando se da la existencia de errores o situaciones que generan conflicto, debido a que es en estas situaciones donde se activan procesos de autorregulación (Lucangeli et al., 2019; Petrova \& Kozarova, 2018) y permiten la autocorrección (Veenman, 2015). Tomando esto en cuenta, al estimular el pensamiento de tipo metacognitivo es importante considerar las siguientes orientaciones:

- Es pertinente que el docente explicite la forma de realizar metacognición (Soto et al., 2019). Por ejemplo, explicitar las etapas de planificación, supervisión, regulación y evaluación mientras se lleva a cabo la actividad pedagógica. Esto se puede concretar por medio de guías o apoyos a la vista, imágenes o logos, verbalizando en qué etapa se encuentran y dando un tiempo específico para su ejecución. Luego de llevarlo a cabo varias veces, los estudiantes comienzan a internalizar el procedimiento y a ganar autonomía.

- Estimular la conciencia de los propios procesos mentales, incentivando al estudiante al uso de auto preguntas como autoinstrucciones (Veenman, 2015), tales como ¿Hice esto? ¿Creo que lo hice bien? ¿Qué podría mejorar? ¿Cumplími meta? ¿Qué dificultades tuve? ¿Cómo las podría solventar para una próxima vez? El uso de auto preguntas da un rol activo al aprendiz y permite que éste organice la información al momento de procesarla. Además, por medio de las auto preguntas se puede incitar a que el estudiante cuestione sus estrategias metacognitivas para mejorarlas progresivamente.

- Orientar el conocimiento de los recursos personales, las propias estrategias y la tarea, por medio del diálogo con el estudiante. Es importante dar espacios en la clase para hablar explícitamente sobre el pensar y sobre cómo lo hacemos, compartiendo experiencias y estrategias entre los aprendices y mediadas por el docente en contexto de aprendizaje colaborativo (Wismath \& Orr, 2015).

- Proponer actividades desafiantes, situaciones problemáticas (Iwai, 2016) y contextualizadas (Wismath \& Orr, 2015), que pongan al aprendiz en un conflicto cognitivo. Este tipo de situaciones estimula niveles de motivación que permiten mantenerse en la tarea hasta que se finalice, y son apropiadas para instalar la verbalización de los pasos mentales o acciones metacognitivas que se han ido haciendo para llevarla a cabo.

- Determinar la progresión de la visibilidad de la metacognición. En una sola clase el aprendiz no logra instalar todas las estrategias ni un nivel de conciencia profundo sobre la propia forma de aprender, ya que se requieren varias semanas para instalarse (Iwai, 2016). Se sugiere partir con actividades breves, con preguntas concretas sobre el pensar y sobre cómo ellos se auto revisan o auto observan. De manera progresiva, enriquecer con más preguntas y más referencias hacia el proceso metacognitivo que se va llevando a cabo.

Para estimular el pensamiento crítico, es importante considerar que el aprendizaje en el contexto educativo ya permite en sí mismo el desarrollo indirecto de las habilidades del pensamiento, pero esto no es una garantía del desarrollo del pensamiento crítico. Asimismo, se pueden considerar ambientes de resolución de problemas y tecnología (Lawless \& Brown, 2015). Es necesaria la estimulación de ciertas habilidades que permitan a los estudiantes cuestionar, valorar y argumentar sus acciones, mejorando la toma de decisiones en diferentes aspectos de la vida académica. 
Algunas sugerencias al respecto, considerando aportes de Ennis (2011) y otros autores, son:

- Poner a los estudiantes en situaciones en las cuales realicen una valoración de las fuentes: si son fidedignas, si son validadas, si el autor sabe del tema, si la fuente es apropiada al contexto de la situación.

- Dar la posibilidad a los estudiantes de que, por medio de estrategias de enseñanza como el debate o la discusión por medio de preguntas (Borjas \& De La Peña, 2009), formulen argumentos con adecuada información, organización y lenguaje para que sea comprensible para el interlocutor.

- Por medio de preguntas o situaciones peculiares, despertar la curiosidad ante la información y la búsqueda de otra que pueda complementarla o servir incluso de contraste.

- En estudiantes con un pensamiento un poco más abstracto, tales como los de educación secundaria, potenciar la reflexión de las ideas de otros autores, para elaborar los propios argumentos.

- Al hacer uso del debate, es importante que el docente incite a valorar del empleo del argumento: si el argumento es pertinente para el cuestionamiento, o si no se relaciona o carece de conexiones con la situación, por ejemplo. Ello puede llevarse a cabo por medio de algunas preguntas (Borjas \& De La Peña, 2009), tales como ¿Esto es apropiado para responder o resolver la situación? ¿La idea o argumento puede mejorarse? ¿De qué forma se puede mejorar para que sea más pertinente? Con ello se podría lograr que se alcance el objetivo de responder apropiadamente a lo planteado y disminuir progresivamente las respuestas desviadas y ambiguas.

- Por medio del uso de casos ficticios, dilemas o problemas (Birgili, 2015) ya resueltos (algunos bien resueltos y otros mal resueltos), se puede trabajar puntualmente la habilidad de persuadir o convencer al interlocutor con razones objetivas, pertinentes y fundamentadas.

- Llevar a cabo actividades o juegos donde se propongan una amplia gama de argumentos a una problemática, en un contexto de resolución de problemas (Birgili, 2015). Luego, solicitar a los estudiantes que los organicen según su prioridad y pertinencia.

- Es muy importante hacer actividades prácticas donde los estudiantes manipulen o tengan experiencias concretas de aprendizaje, donde indaguen y experimenten, por ejemplo: aprender a buscar y usar fuentes fidedignas, valoración del conocimiento validado $\mathrm{v} / \mathrm{s}$ conocimiento ordinario.
En fuentes online (Lawless \& Brown, 2015), por ejemplo, comparar información de un blog con información de un libro digital.

- Provocar a los aprendices para elaborar de forma individual como colectiva los argumentos y el relato para la persuasión. Se puede incitar por medio de situaciones desafiantes o preguntas específicas que generen un conflicto cognitivo, por ejemplo: dar información errada y que ellos se percaten de ello, dar una situación problema estimulando como desafío su resolución (Birgili, 2015), entre otros.

- En las actividades debe intencionarse una etapa final, donde se dé la gestación de conclusiones propias, objetivas, y se cuestione sobre su transferencia a la realidad por medio de preguntas (Borjas \& De La Peña, 2009) como: ¿En qué situación de mi vida diaria podría emplear esto? ¿Cómo lo puedo ajustar? ¿Qué utilidad tiene?

La estimulación del pensamiento creativo, como se señaló anteriormente, debe ir de la mano con experiencias de aprendizaje que presenten dilemas, desafíos, problemas reales cotidianos o disyuntivas que requieran de propuestas o soluciones que optimicen los recursos, el tiempo, el espacio. Considerando a Rawlinson (2017), De Bono (2012) y a otros autores, se sugiere:

- El planteamiento de un problema o situación a resolver que esté bien caracterizada o se presente de forma específica al estudiante (Birgili, 2015). Además, la situación debe ser significativa para el aprendiz: pueden ser situaciones de su entorno pero aún desconocidas para él. Pueden presentarse mediante: videos con dilemas, problemas reales sin resolver o ya resueltos, noticias (por ejemplo: sobre contaminación, optimización de recursos, problemas reales en la ciudad), entre otros.

- El docente debe plantear el problema como un desafío (Birgili, 2015), que pone a prueba las capacidades personales y que es posible de solventar con las estrategias y recursos personales de los aprendices.

- Llevar a cabo verbalizaciones, en la cual se realice una valoración objetiva del conflicto o situación al que se enfrentan los estudiantes. Por ejemplo, hacer preguntas sobre las fortalezas y debilidades, barreras, (por ejemplo, análisis FODA) que posteriormente dé luces de posibles vías de solución o propuestas.

- Explicitar que se debe realizar una priorización de los problemas o conflictos según su nivel de urgencia o de progresión para ser resueltos (De Bono, 2012). Por ejemplo, en una situación 
problema o dilema se pueden dar varias barreras o conflictos de deben ser localizados por los estudiantes. Una vez identificados, se solicita que los organicen por prioridad o por orden de progresión apropiada para ser resueltos.

- Se debe abrir discusión en el aula, propendiendo a la generación de alternativas variadas como posibles caminos para solucionar las situaciones propuestas (Rawlinson, 2017). Se puede hacer por medio de lluvia de ideas que se organizan en el pizarrón, o que lo elaboren de forma grupal y la síntesis final se organiza en el pizarrón para crear una macro red de ideas que los mismos estudiantes originaron. Esto ayuda a que los aprendices visualicen de manera más concreta las ideas y propuestas, vinculándolas entre sí

- Es trascendental que se insista como docente en que los mismos estudiantes evalúen si las estrategias o propuestas que plantean realmente son novedosas $u$ originales, puesto que es un componente fundamental del pensamiento creativo. Además, evaluar su utilidad en el contexto de la problemática o dilema presentado para resolver.

- Llevar a cabo actividades en que se haga empleo de otras vías o estrategias ya existentes y conocidas pero en un contexto nuevo, ajustándose a la situación (modificación contextual) (De Bono, 2012).

- Las actividades siempre deben intencionar el uso eficiente de recursos orientado hacia la economía de materiales, tiempo, ambiente y personas; o el uso de oportunidades que garantizan la economía en estos aspectos (De Bono, 2012). Se puede dialogar con los aprendices respecto de ello al finalizar la actividad, como cierre de ésta.

- Hacer preguntas (Borjas \& De La Peña, 2009) sobre la valoración de la estrategia o solución, y del proceso de búsqueda de ella, de cara a futuros procedimientos (qué se mantendría del proceso efectuado, qué se suprimiría, qué se ajustaría).

- Impulsar que las actividades a desarrollar permitan la interacción social entre aprendices, pues el pensamiento creativo es un proceso determinado por interacciones sociales y situaciones (Loi \& Dillon, 2006).

\section{CONCLUSIONES}

El pensamiento, como se expuso a lo largo de este artículo, conlleva procesos mentales complejos e integrados, que deben ser considerados en los contextos educativos y en sus diversos niveles. En el caso del pensamiento metacognitivo, crítico y creativo los aportes desde la psicología y los más recientes desde las neurociencias revelan que son procesos mentales muy elaborados que tienen implicancia en el aprendizaje y rendimiento académico.

Cada uno de estos tipos de pensamiento debe estimularse de forma integral, en contextos reales y en situaciones de aprendizaje que permitan el constante diálogo con los aprendices (Wismath \& Orr, 2015). Es trascendental que se estimulen de forma explícita (Lawless \& Brown, 2015) sin esperar su desarrollo de forma intuitiva por parte del aprendiz, teniendo en cuenta que las actividades deben tener cierta frecuencia y consistencia para que se instalen los procesos (Birgili, 2015), pues si son actividades aisladas se diluye esta construcción cognoscitiva en el tiempo. Asimismo, es relevante que se estimule el pensamiento y con ello el aprendizaje en contextos sociales (Wismath \& Orr, 2015), pues son las situaciones en las que, por medio de las interacciones, se construyen las representaciones mentales y se desarrollan los procesos mentales superiores.

Todos estos aspectos permiten a los profesionales que trabajen en la educación centrarse en el alcance del aprendizaje de los estudiantes y no solo en la ejecución de la enseñanza. Esto permitiría un mayor desarrollo del pensamiento en los estudiantes para dar soluciones a situaciones reales y no solo la adquisición de información por medio de una instrucción reproductiva. De esta manera se logrará generar una construcción progresiva del aprendizaje autónomo y autorregulado en los aprendices, y se propenderá a un adecuado desarrollo integral como personas.

\section{REFERENCIAS}

Abraham, A. (2018). The neuroscience of creativity. Cambridge: Cambridge University Press.

Aguirre, L. (2016). Evaluación de una propuesta para el desarrollo de la escritura en estudiantes universitarios a partir de habilidades de metacognición. Logos (La Serena), 26(2), 181-196.

Beaty, R. E.; Benedek, M.; Silvia, P. J.; Schacter, D. L. (2016). Creative cognition and brain network dynamics. Trends in Cognitive Sciences, 20(2), 87-95.

Beaty, R. E.; Kenett, Y. N.; Christensen, A. P.; Rosenberg, M. D.; Benedek, M.; Chen, Q.; Fink, A.; Qiu, J.; Kwapil, T.; Kane, M. J.; Silvia, P. J. (2018). Robust prediction of individual creative ability from brain functional connectivity. Proceedings of the National Academy of Sciences, 115, 1087-1092.

Belmonte, V. (2013). Inteligencia emocional y creatividad: factores predictores del rendimiento académico. Disertación Doctoral, Universidad de Murcia, España.

Birgili, B. (2015). Creative and critical thinking skills in problema-based learning environments. Journal of Gifted Education and Creativity, 2(2), 71-80.

Borjas, M.; De La Peña, F. (2009). Desarrollo de habilidades 
de pensamiento creativo en el área de Ciencias Naturales y Educación Ambiental. Zona Próxima, 10, 12-35.

De Bono, E. (2012). Seis Sombreros para Pensar. Barcelona: Paidós.

Dietrich, A. (2004). The cognitive neuroscience of creativity. Psichonomic Bulletin \& Review, 11(6), 1011-1026.

Elder, L.; Paul, R. (2008). Critical thinking: strategies for improving student learning. Journal of Developmental Education, 32(1), 32-33.

Ennis, R. (2011, mayo). The nature of critical thinking: An outline of critical thinking dispositions and abilities. Trabajo presentado en el Sixth International Conference on Thinking at MIT, Cambridge, Inglaterra.

Flaherty, A. (2005). Frontotemporal and dopaminergic control of idea generation and creative drive. The Journal of Comparative Neurology, 493(1), 147-153.

Fleming, S.; Dolan, R. (2012). The neural basis of metacognitive ability. Philosophical Transactions of the Royal Society B: Biological Sciences, 367(1594), 1338-1349.

Gormley, W. T. (2017). The critical advantage: Developing critical thinking skill in school. Cambridge, Massachusetts: Harvard Education Press.

Green, A. E.; Cohen, M. S.; Raab, H. A.; Yedibalian, C. G.; Gray, J. R. (2015). Frontopolar activity and connectivity support dynamic conscious augmentation of creative state. Human Brain Mapping, 36(3), 923-934.

Huber, C. R.; Kuncel, N. R. (2016). Does College Teach Critical Thinking? A Meta-analysis. Review of Educational Research, 86(2), 431-468.

Iwai, Y. (2016). Promoting strategic readers: Insights of preservice teachers' understanding of metacognitive reading strategies. International Journal for the Scholarship of Teaching and Learning, 10(1), 1-7.

Jiménez-Aleixandre, M. P. (2010). 10 Ideas Clave. Competencias en Argumentación y Uso de Pruebas. Barcelona: Graó.

Karbalaei, A. (2011). Metacognition and Reading comprehension. Íkala, Revista de Lenguaje y Cultura, 16(28), 5-14.

Kim, K.H. (2011). The creativity crisis: The decrease in creative thinking scores on the Torrance Tests of Creative Thinking, Creativity Research Journal, 23(4), 285-295.

Lacon, N.; Ortega, S. (2008). Cognición, metacognición y escritura. Revista Signos, 41(67), 231-255.

Lawless, K. A.; Brown, S. W. (2015). Developing scientific literacy skills though interdisciplinary, tecnology-based global simulations: GlobalEd 2. The Curriculum Journal, 26(2), 268-289.

Loi, D.; Dillon, P. (2006). Adaptive educational environments as creative spaces, Cambridge Journal of Education, 36(3), 363-381.

López, O.; Navarro, J. (2008). Estudio comparativo entre medias de creatividad: TTCT vs. CREA. Anales de Psicología,
24(1), 138-142.

Lucangeli, D.; Fastame, M. C.; Pedron, M.; Porru, A.; Duca, V.; Hitchcott, P. K.; Penna, M. P. (2019). Metacognition and errors: the impact of self-regulatory trainings in children with specific learning disabilities. ZDM Mathematics Education, 51(4), 577-585.

Madore, K. P.; Jing, H. G.; Schacter, D. L. (2016). Divergent creative thinking in young and older adults: Extending the effects of an episodic specificity induction. Memory \& Cognition, 44(6), 974-988.

Mihov, K.; Denzler, M.; Förster, J. (2010). Hemispheric specialization and creative thinking: A meta-analytic review of lateralization of creativity. Brain and Cognition, $72(3), 442-448$.

Molenberghs, P.; Trautwein, F.M.; Böckler, A.; Singer, T.; Kanske, P. (2016). Neural correlates of metacognitive ability and of feeling confident: a large-scale fMRI study. Social Cognitive and Affective Neuroscience, 11(12), 1942-1951.

Monereo, F. (2001). Ser estratégico y autónomo aprendiendo. Unidades didácticas de enseñanza estratégica. Barcelona: Graó.

Moreno, W.; Velázquez, M. (2017). Estrategia didáctica para desarrollar el pensamiento crítico. Revista Iberoamericana sobre Calidad, Eficacia y Cambio en Educación, 15(2), 53-73).

Nieto, A.; Saiz, C. (2011). Skills and dispositions of critical thinking: are they sufficient? Anales de Psicología, 27(1), 202-209.

Ospina-Rave, B.; Brand-Monsalve, E.; Aristizabal-Botero, C. (2017). Development of a measurement index of critical thinking in professional formation. Investigación y Educación en Enfermería, 35(1), 69-77.

Pacheco, V. (2003). La inteligencia y el pensamiento creativo: Aportes históricos en la educación. Revista Educación, 27(1), 17-26.

Perchtold, C. M.; Papousek, I.; Koschutnig, K.; Rominger, C.; Weber, H.; Weiss, E. M.; Fink, A. (2018). Affective creativity meets classic creativity in the scanner. Human Brain Mapping, 39(1), 393-406.

Petrova, G.; Kozarova, N. (2018). Concept of working with mistakes in the education process. Ad Alta: Journal of Interdisciplinary Research, 8(1), 148-153.

Prieto, M. D.; López, O.; Ferrándiz, C.; Bermejo, M. R. (2003). Adaptación de la prueba figurativa del Test de Pensamiento Creativo de Torrance en una muestra de alumnos de los primeros niveles educativos. Revista de Investigación Educativa, 21(1), 201-213.

Rawlinson, J. G. (2017). Creative thinking and brainstorming. New York: Routledge.

Shimamura, A. (2000). Toward a cognitive neuroscience of metacognition. Consciousness and Cognition, 9, 313-323.

Sinatra, G. M.; Taasoobshirazi, G. (2018). The self-regulation of learning and conceptual change in science: Research, 
theory, and educational applications. In Schunk, D. H.; Greene, J. A. (Eds.), Handbook of self-regulation of learning and performance (pp. 153-165). New York: Routledge.

Soto, C. (2002). Metacognición, Cambio Conceptual y Enseñanza de las Ciencias. Bogotá: Didáctica Magisterio.

Soto, C.; Gutiérrez de Blume, A. P.; Jacovina, M.; McNamara, D.; Benson, N.; Riffo, B. (2019). Reading comprehension and metacognition: The importance of inferential skills. Educational Psychology \& Counselling, 6, 1-20.

Tanikawa, H. A. M.; Boruchovitch, E. (2016). Monitoramento metacognitivo de alunos do ensino fundamental. Psicologia Escolar e Educacional, 20(3), 457-464.

Tenías, M. (2012). Pensamiento crítico en estudiantes de educación, mención castellano y literatura. Revista Trilogía, 6, 93-104.

Veenman, M. V. J. (2015). Metacognition. In Afflerbach, P. (Ed.), Handbook of individual differences in Reading (pp. 26-40). New York: Routledge.

Wismath, S. \& Orr, D. (2015). Collaborative Learning in Problem Solving: A case study in metacognitive learning. Canadian Journal for the Scholarship of Teaching and Learning, 6(3), 1-17.

Esta publicación se realizó en el marco del proyecto E06/19 “Aprendizaje autorregulado de profesores en formación de Pedagogía en educación diferencial de dos Universidades públicas chilenas”, adjudicado por medio del Concurso Interno de Investigación Científica y Tecnológica en Educación, de la Universidad de Los Lagos, Chile.

Recibido: 13 de marzo de 2019 Aprobado: 16 de diciembre de 2019 